assessment. The combination of a distressed restless patient and a stomach containing a large volume of blood may decrease the chances of accurately identifying the cause of bleeding. In these circumstances it is usually easier to assess the antrum and duodenal cap. If these are clear, attention should then be directed at the upper half of the stomach. A useful manoeuvre in these circumstances is to dilate the stomach with air when small lesions may become apparent. The diagnostic return more than balances the slight extra risk involved. Experience in Blackpool suggests that in haemorrhage associated with multiple gastric erosions which is recurrent or persistent, dilatation of the stomach may show that bleeding occurs from one minute lesion, usually high in the stomach and involving a submucosal artery. If endoscopic coagulation therapy is not available or not successful, early surgery is the best treatment.

F I LEE

Department of Gastroenterology,

Victoria Hospital,

Blackpool FY8 3NR.

\section{Biphasic hepatitis in HBV/HDV coinfected parenteral drug abusers}

SIR,-We would like to refer to a few of the issues raised by Dr Govindarajan and colleagues (Gut 1986; 27: 19-22). We agree with the authors that delta (HDV) infection is a cause of severe relapse of acute hepatitis B. ${ }^{12}$ To assess the magnitude of the problem, we have determined the prevalence of biphasic hepatitis in a large series of parenteral drug abusers admitted to our infectious diseases clinic between January 1979 and December 1983 for acute HBV/HDV coinfection or classical hepatitis B. Biphasic hepatitis was significantly more frequent in patients with delta coinfection $(53 / 180 ; 29.4 \%)$ than in those with classical hepatitis B (13/98; $13 \cdot 3 \%)(p<0.01)$. In agreement with Dr Govindarajan, a significant proportion of delta infected patients $(10 / 53 ; 18.9 \%)$ had severe relapses (prothrombin activity $<50 \%$ the level in normal controls) with a fulminant course (and death) in two of these patients. On the contrary, the relapses we have observed in patients with classical hepatitis B generally had a mild course. We would like to discuss two more problems. One regards the time of occurrence of relapses in HBV/HDV coinfected patients. The authors report patients with a second peak of alanineaminotransferase within 30 days from the initial episode. We would emphasise that the relapse of acute $\mathrm{HBsAg}$ positive hepatitis serologically associated with HDV infection may be present in some patients (six in our series) during the second month, when the patients are still
HBsAg positive, but usually discharged from the hospital. One can argue these might be considered cases of HDV superinfection. The detection of serum $\delta \mathrm{Ag}$ in four of these during the first acute episode, as well as the uneventful course of the disease in all the subjects, are against this hypothesis. ${ }^{34}$ Lack of follow up in parenteral drug abusers with acute HBV/HDV coinfection may produce an underestimate of the prevalence of relapses. Because of the possible asymptomatic course of the relapse, this error might be increased. We think that parenteral drug abusers with acute HBsAg positive hepatitis must be followed up with frequent clinical, biochemical, and serological tests until clearance of $\mathrm{HBsAg}$.

The other problem regards the serological diagnosis of HDV infection in patients with acute (primary) hepatitis B virus infection. As anti- $\delta$ antibody in patients previously negative for serological delta markers is sometimes detectable only four to six weeks after the onset of the disease, ${ }^{3}$ the incidence of HDV infection, and thus the risk of severe hepatitis, might be underestimated if serial serum samples are not tested.

Regarding the question put by the authors about the possible development of chronic $\mathrm{HBsAg}$ positive hepatitis in patients with HBV/HDV coinfection with a biphasic course of the acute disease, our data show that, apart the occurrence of severe (and fulminant) hepatitis, these patients are not at risk of developing chronic hepatitis. All the patients who survived the acute episode (and the relapse) cleared $\mathrm{HBsAg}$ and seroconverted to anti-HBs.

F CAREDDA, S ANTINORI, RE T, C PASTECCHIA, AND M MORONI.

Infectious Diseases Clinic, University of Milan,

Osp L Sacco,

20157 Milan,

Italy.

\section{References}

1 d'Arminio Monforte A, Caredda F, Rossi E, Lopez S, Moroni M. Delta system in HBsAg-positive drug addicts with hepatitis. In: Szmuness W, Alter HJ, Maynard JE, eds. Viral hepatitis. 1981 International Symposium. Philadelphia: the Franklin Institute Press, 1982: 729.

2 Caredda F, d'Arminio Monforte A, Rossi E, Farci P, Smedile A, Tappero G, Moroni M. Prospective study of epidemic delta infection in drug addicts. In: Verme G, Bonino F, Rizzetto M, eds, Viral hepatitis and delta infection. New York: Alan R Liss, 1983: 245-50.

3 Caredda F, Orlando G, Antinori S, Re T, Zampini L, Moroni M. Simultaneously acquired hepatitis B and hepatitis D virus infections. $B r$ Med $J$ 1985; 291: 51-2. 
4 Caredda F, Rossi E, d'Arminio Monforte A, et al. Hepatitis B virus-associated coinfection and superinfection with $\delta$ agent: indistinguishable disease with different outcome. $J$ Infect Dis 1985; 151: 925-8.

\section{Books}

Stress and common gastrointestinal disorders: a comprehensive approach By G Dotevall. (Pp. 172; illustrated; $£ 35.00)$. New York: Praeger Publishers, 1985.

It is the author's belief that duodenal ulcer disease, non-ulcer dyspepsia, irritable bowel syndrome, and functional constipation are '. . . stress-related to the extent that emotional factors are important in their pathogenesis.' Few gastroenterologists would dispute this proposition, but in this contentious field, multi-author books reveal confusion rather than consensus. As Grant Thompson showed in The irritable gut, a monograph on this subject has the virtues of consistent conceptual structure and literary style, while leaving room for another point of view. The latter is now provided by Gerhard Dotevall, a distinguished Swedish contemporary.

His approach to the problem was summarised by Engel: 'The dominant model of disease is biomedical, with molecular biology its basic scientific discipline. It assumes disease to be fully accounted for by deviations from the norm of measureable (biological) variables. It leaves no room within its framework for the social, psychological and behavioural dimensions of illness.' Those who doubt the dominance of the biomedical model have only to scan the pages of this and many other journals, and those who are insistent upon its adequacy probably suffer from inadequate exposure to patients (it's odd, when you think about it, that most of the papers published in medical journals have little or no relevance to the practice of medicine). Dotevall prefers the 'biopsychosocial model'; while this might be cynically described as the biomedical model with added fudge factors, so is life.

The problem facing the author was the construction of clear concepts from a mass of data which is both contradictory and inadequate. As was inevitable, he was not altogether successful. His review of the relation between the CNS and the gut is inadequate, and ignores recent advances in understanding the enteric nervous system and the predominantly afferent role of the vagus, while the brief consideration of peptides is unhelpful. $\mathrm{He}$ is on much firmer ground in his consideration of the nature of stress and the functional syndromes, and rightly stresses the overlap between these syndromes. The main support for his concepts derives from his own studies on the efficacy of psychotherapy in irritable bowel syndrome and duodenal ulcer disease, and certainly this work deserves to be more widely recognised. It is therefore surprising to find considerable emphasis on the pharmacological management of these disorders, the more so since it is generally acknowledged that with the exception of ulcer disease, drugs are often little better than placebos.

This book stimulates by challenging some accepted dogma, and it informs by citing relevant work that is often not widely known. The references are carefully selected and appropriate rather than obsessionally comprehensive. Gastroenterologists will profit from reading this book. The gain will be intellectual rather than financial; at $£ 35.00$ for 172 pages, the publishers seem to be heading for some sort of inflationary record.

DAVID WINGATE

Disorders of the small intestine Edited by $\mathrm{C} \mathrm{C}$ Booth and G Neale. (Pp. 438; illustrated; £32.50). Oxford: Blackwell Scientific Publications, 1985.

It is high time for a systematic book devoted to disorders of the small intestine. This book fills that gap. It is multi-author and international, though largely London based.

We are told that 'The book is intended primarily for postgraduate students and for practising gastroenterologists ...' What do we require of such a volume? We need it to be authoritative, relatively comprehensive and at a high level. Physicians will look here for advice as to what to do when standard investigation has not given the answer or when standard therapy has not given the desired result and also for conditions not in the general textbooks. How does this volume measure-up? In most things, very well indeed. For a common condition, such as Crohn's disease, there is a very good description of the pathology and clinical features: diagnosis and differential diagnosis are helpfully discussed, and management is well categorised according to the state of the patient. For a rare condition, such as Whipple's disease, there is a lucid description of the pathology and of the heterogeneous clinical features, with a clear discussion of treatment.

There are, however, parts which could be improved in future editions. In such a volume it is surely unnecessary to state elementary points such as 'Diagnostic investigations should be chosen with care ...' or that 'It is always important to inquire into the patient's previous history'. For a book 